\title{
Smart-Nanobots: The Future of HIV AIDS Treatment
}

\author{
Sabyasachi Choudhuri ${ }^{1}$, Dr. Soumya Deb ${ }^{2}$ \\ ${ }^{1}$ B. Pharm Final year student, Institute of Pharmacy, Jalpaiguri 735101, West Bengal, India \\ ${ }^{2}$ M. Pharm, PhD, Department of Pharmacognosy, Institute of Pharmacy, Jalpaiguri 735101, West Bengal, India
}

\begin{abstract}
In India, more than 50 lakhs of people are infected by AIDS. There is currently no medicine that can cure AIDS. The drugs used are only able to increase the lifespan of the patient for a few years only. Also, during the last decade, the use of artificial intelligence tools in nanotechnology research has increased widely. In this paper, we are going to present a theoretical concept of the current trend and future perspective in the development of Nanobots for treatment of HIV AIDS. Nanobots can be injected or transferred inside the body to treat complex diseases. To make the treatment more specific we can use nanobots to sense AIDS-infected WBCs.
\end{abstract}

Keywords: Nanotechnology, Robotically enhanced Organism, Nano-biosensor, Nanochip, Artificial Intelligence, Machine Learning, Artificial Neural Network

\section{Introduction}

Nanorobotics is the study of robots using nanotechnology. These are robots of size a few nanometres. Nanobots consist of 'Nano' and 'Bots'. Nano means one billionth of one and bot is the short form of a robot which is a machine that may be programmed to perform a specific task. In the near future, we would be having nanoscopic bots that crawl or swim inside our blood without any allergic reaction. Nanobots can be programmed to target the infected WBCs precisely thereby decreasing the chance of allergic reactions. This may be termed as REOs (Robotically Enhanced Organisms). We could even use nanobots inside our body to stream inside environment to a wireless monitoring system as cyborgs in sci-fi movies. This concept of Nanotechnology was first proposed by late noble physics Richard P. Feynman in 1959. In his lecture, "There's plenty use of the room at the bottom" [1], Feynman explored the technology for making materials at the scale of nanometres. He said, "A friend of mine Albert $\mathrm{R}$ Hibbs suggests a very interesting possibility of nanomachines. He said to me that, although it is a very wild idea, it would be interesting in surgery if you could swallow the surgeon."

\section{Necessity of Nanotechnology}

Nanotechnology is very much needed because in future we will have the disease which cannot be treated with normal medicine. The nano advantage is that we can create small devices in nano size that can be injected inside our body without causing much pain and stay inside the body and can repair and heal the respective required unhealthy cells and can easily destroy them if required. This will enable nanobots to get inside the cells and monitor cells within the living body.

\section{AI/ML implementation in Nanobots}

Bridging the link between current nanotechnology and
Artificial Intelligence can boost research and provide a new generation of bioinformatics that will have a large impact on our society's health. This would probably provide the means so that nanotechnology, biology and computer merge together for the betterment of society. AI when joined with nanorobots can supplement the gap within the immune system. There are several reasons why AI paradigms are used in nano research.[2] Nanotechnology suffers the physical limitation of its working scale, where this physical world is completely different from the macroscopic world We need to imagine the correct interpretation of results obtained from any system or device. It is in this context that AI tools such as machine learning can play a role in getting scientific results as well as the development of future nano applications.

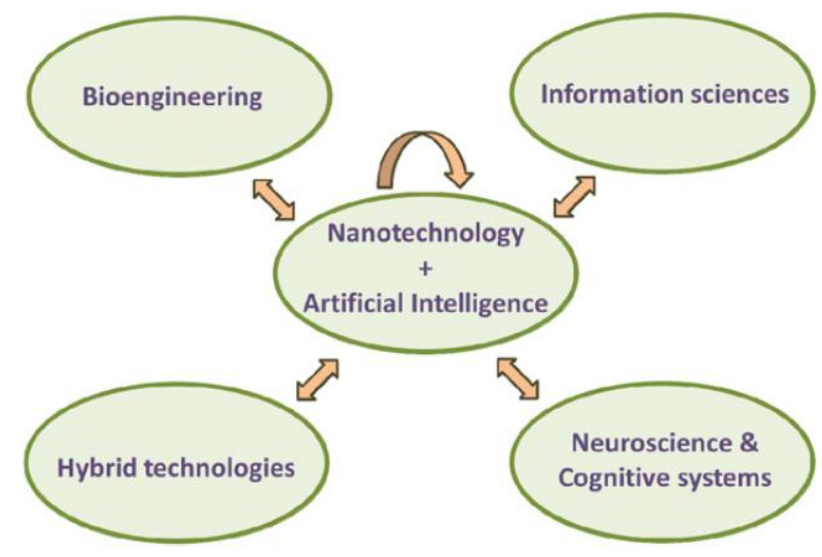

Figure 1: Technologies linked

Machine learning methods include approaches like artificial neural network (ANNs), a set of interconnected nodes whose connection are determined through a supervised or unsupervised algorithm. This is used for learning inputoutput functions. Another type of bio-inspired AI model based on evolutionary or genetic algorithms also provide the solution to optimization and search problem. There are many machine learning approaches that use single or combined methods including support vector machines, Bayesian

Volume 9 Issue 6, June 2020 


\section{International Journal of Science and Research (IJSR) \\ ISSN: 2319-7064}

ResearchGate Impact Factor (2018): 0.28 | SJIF (2019): 7.583

networks, decision trees, etc, which can be applied to complex classification, clustering, association, prediction, data mining and control problems [3] in nanotechnology research. Nanotechnology deals with building things bottomup, while AI typically provides a top-bottom approach to solve the problem. This combination will lead to a solution for many complex problems that require multiple description levels and interactions.

AI can be implemented in Nanorobotics to supplement the human immune system by following ways:

- An AI program should be capable of processing necessary information to act within the human body.

- The AI should be trained by uploading information about the expected chemical and molecules of the human body. The AI model should contain detailed information about the human body, cell types, chemical types, concentration, location and physiological relations.

- This immense library of knowledge would be required to enable the AI-enabled machine to recognize the difference between the normal cells and an infected cell.

- Unsupervised machine learning model can enable AI bot to continuously learn without human interference using methods such as cluster analysis.

- With enough training the AI program should be able to create new programs and respond effectively to unseen categories of AIDS and HIV.

- Successful unsupervised learning can help in identifying antagonists and at the same time ignore the healthy cells

\section{Concepts of Nanorobots (Nanobots)}

Nanorobotics is a major field of nanotechnology. The Czech word "robota" stands for 'work'. Thus, a Nanorobot is often seen as nothing else than a nanoscale machine that does some work. [4] Nanorobotics is a field that consists of efforts of physicists, chemists, biologists, computer scientists, and engineers. Nanorobotics is a convergence of technologies [5].

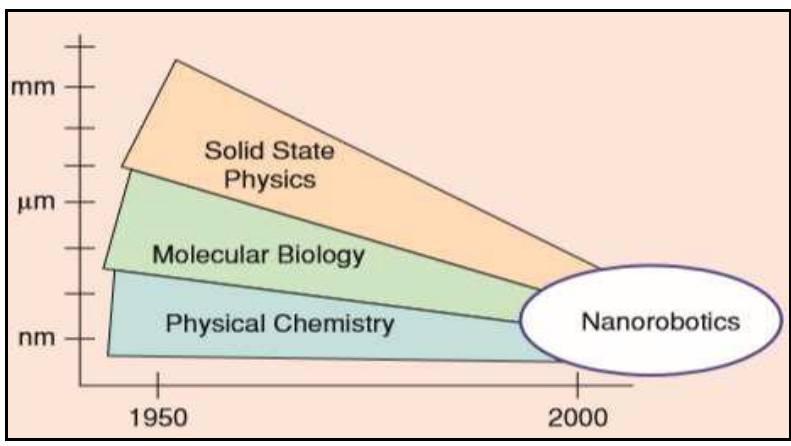

Figure 2: Scope of Nanorobotics

The ideal characteristics of Nanorobots include:

- They have a size range in between 0.5 to 3 microns large with 1-100 nm parts.

- They have a passive diamond exterior which prevents itself from being attacked by the immune system.

- It will work on a carrier wave frequency of 1-100 MHz.

- It might be able to make multiple copies of itself to replace worn-out units by self-replication.

- It should possess swarm intelligence- decentralized artificial intelligence.
- It should contain nano information processors - for programming and controlling nanobots. [6]

The advantages of Nanorobots are:

- Operates autonomously.

- Protects the human body against the pathogen.

- Rapid treatment of disease.

- They are durable and can be kept operational for a long period of time

- It is of very tiny size so it can easily flow in the body without blocking the capillary flow.

- Minimal or no post-treatment care is required.

The disadvantages of Nanobots are:

- The initial design is very expensive.

- The design is a very complicated one.

- Sometimes it is difficult to navigate to the target organ.

- Precise actuation is a major challenge here.

\section{Advantages of Nanobots over conventional medicines}

Nanobots are very much smart, robust and precise while treating any disease. While traditional medicines are less efficient. The bioavailability of any drug is a major problem. The conventional medicines cannot be guided from outside. These are the conditions where Nanobots comes into the scenario.

\section{Structure of Nanobot}

The design of Nanobot is the most important. As the matter it is in nanoscale, it behaves completely different from the larger level. The Nanobots here we are describing will be freely floating inside the body exploring and detect the HIV virus. The Nanobots consists of four main part:[7]

1. DNA sensor

2. CPU

3. RNA converter

4. Power System

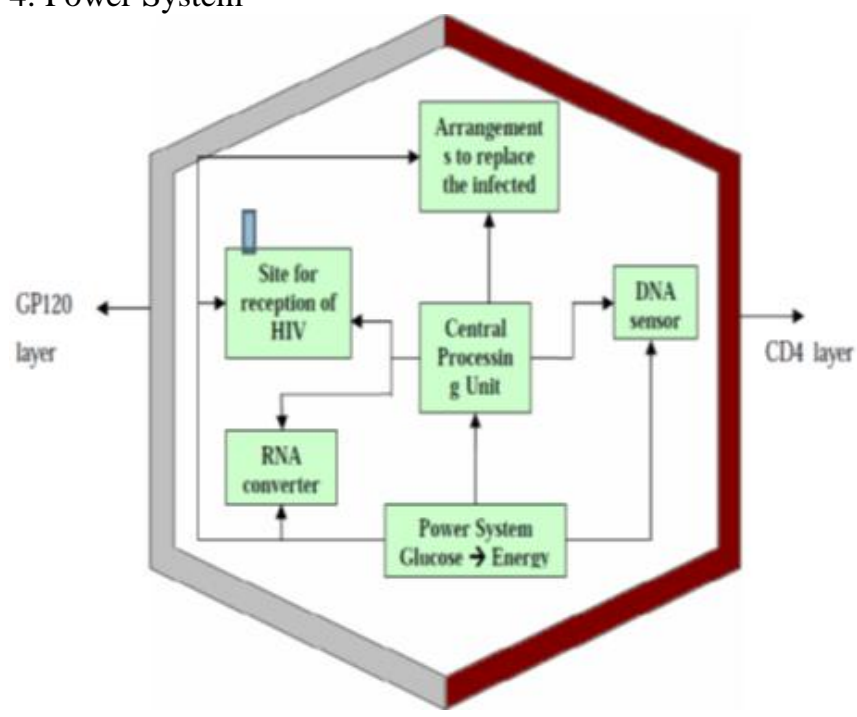

Figure 3: Structure of a Nanobots

The factors which should be kept in mind while designing them are as follows: 


\section{International Journal of Science and Research (IJSR) \\ ISSN: 2319-7064}

ResearchGate Impact Factor (2018): 0.28 | SJIF (2019): 7.583

- Technique used: Bottom-up approach is involved in assembling structure atom by atom or molecule by molecule.

- Size: They are typically 0.5 to 3 microns large with 1-100 parts. Three microns is the highest limit for any nanorobots to build because above this will block capillary flow.

- Structure: The structure of nanorobot can be divided into two parts:

- Interior: Closed, vacuum environment into which a liquid from outside normally cannot enter unless it is needed for chemical analysis.

- Exterior: Subjected to various chemical liquid in our bodies.

- Chemical elements: Carbon is the principal element comprising the bulk of a medical nanorobot, probably in form of diamond or diamondoid/fullerene nanocomposites. These are used largely because of the tremendous strength and chemical inertness of diamond [8]. Light elements such as hydrogen, sulphur, oxygen, nitrogen, fluorine, silicon etc. may also be used.

- Defending immune system: The ideal exterior is a passive diamond. Several experimental studies show that the smooth flawless surface of the diamond will show less leukocyte activity and less fibrinogen absorption.

- Power source: The bot should be able to metabolise local glucose and oxygen for energy. There are literally numerous power sources that are available inside the human body.

- Communication: The nanobots should be able to receive broadcast type messages into the body (acoustic messaging) for the proper functioning of the nanobots [8].

- Tracking: The exact location should be available to receive high position accuracy of its location in a navigation network.

\section{Working procedure of Nanobots}

The use of Nanobot is for making the treatment more specific. We will use the Nanodevices that use Nano biosensor holding antibodies for the Ag gp41 \& gp120 (HIV $\mathrm{Ag}$ ) will be tagged to the surface. So, whenever it comes in contact with an infected cell the $\mathrm{Ab}$ will react with it by immunochemical reaction and will identify the infected cell. Nanochip will receive the signal from its sensor to perform the job. Nanotube, on receiving a positive signal gets injected into the cell of the nucleus by nanochip. Nano container will contain highly concentrated DNase and RNase enzyme which can be delivered to the infected cell and can cleave the entire genomic DNA into single nucleotides. These Nanobots responds to acoustic signals, so, Nanobots streaming in the blood accumulates in that place to perform exponentially to kill the infected cell. Thus, virulence is lost. AIDS-infected WBC's are then converted back to the original WBC's. This process will lead to a constant level of WBC's in the bloodstream. This is operated at specific sites and has no side effects. Thus, AIDS patient is provided with an immune system so that he/she defend himself/herself from the disease. These nanobots can be connected over the internet to know the progress of treatment and this data can be used for Machine learning technique for building better products. With the emerging new Nanotechnology and Computer Science with Artificial Intelligence, this killer disease AIDS can also be made in the control of Humans. $[2,3,9]$

\section{Basic Equation}

The basic equation for the conversion of the HIV infected WBC's back to its original form is given below. The host system is the WBC and is converted by the HIV into an infected WBC. This is the reason for AIDS.[10]

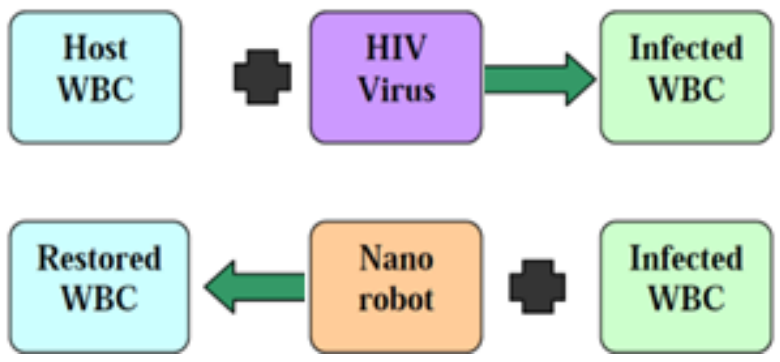

Figure 4: Basic equation

\section{Conclusion}

This paper is merely a theoretical justification. But in recent times due to advancement in the field of nanotechnology and its use in medical science, it gives hope to the new era for the effective use of this technology. These nanobots are a theoretical approach if can be made practical in the near future, then killer disease like AIDS can also be made in control with this emerging new technology. This paper is introducing us to the world of AI and nanotechnology and its importance recognised by various medical studies and technocrats. This is the beginning of nano-era and artificial intelligence and we could expect further improvements and enhancements such as a medicine to AIDS and other diseases using nanotechnology which are not possible through conventional medicine practices.

\section{References}

[1] Feynman RP (1960) Eng Sci CalTech 23.

[2] Mitchell T M 1997 Machine Learning (Boston, MA: McGraw-Hill)

[3] Bishop C M 2006 Pattern Recognition and Machine Learning (Singapore: Springer)

[4] D. J. Wilson, - Nanorobotic devices, October 2018

[5] L. Dong and B. J. Nelson, - Robotics in the small, Part II: Nanorobotics, IEEE Robotics \& Automation Magazine, September 2007

[6] K. S. Sarath, P. N. Beena, and A. Elessy, -Nanorobots a future device for diagnosis and treatment, Journal Pharm Pharmaceutics, vol. 5, no. 1, 2018

[7] P. Khulbe, -Nanorobots: A Review, International Journal of Pharmaceutical Sciences and Research, vol.. 5, no. 6, 2014

[8] K. M. Poornima et al., -Bio-nanorobotics: The milestone of nanotechnology and medicine, 


\section{International Journal of Science and Research (IJSR) \\ ISSN: 2319-7064}

ResearchGate Impact Factor (2018): 0.28 SJIF (2019): 7.583

International Journal of Advanced Research in Computer Science, vol. 5, no. 6, July-August 2014

[9] Brief Review on Nano Robots in Bio Medical Applications, M Sivasankar and RB Durairaj

[10] Anti-HIV Using Nanorobots; Dr. Ankita Joshi et al, IOSR Journal of Electrical and Electronics Engineering (IOSR-JEEE), Volume 7, Issue 6 (Sep. - Oct. 2013).

Volume 9 Issue 6, June 2020

www.ijsr.net

Licensed Under Creative Commons Attribution CC BY 